1 Nonreactive testing

RUNNING HEAD: EVALUATING THE EFFECT OF WITHHOLDING

FEEDBACK

Nonreactive testing: Evaluating the effect of withholding feedback in predictive

learning

Jessica C. Lee

Mike E. Le Pelley

Peter F. Lovibond

UNSW Sydney

Please address correspondence to:

Jessica Lee (jessica.lee@unsw.edu.au)

Acknowledgements:

This study was funded by an Australian Research Council Discovery Grant (DP190103738) awarded to Peter Lovibond. 


\begin{abstract}
Learning of cue-outcome relationships in associative learning experiments is often assessed by presenting cues without feedback about the outcome and informing participants to expect no outcomes to occur. The rationale is that this "no-feedback" testing procedure prevents new learning during testing that might contaminate the later test trials. We tested this assumption in four predictive learning experiments where participants were tasked with learning which foods (cues) were causing allergic reactions (the outcome) in a fictitious patient. We found that withholding feedback in a block of trials had no effect on causal ratings (Experiments 1 and 2), but it led to regression towards intermediate ratings when the missing feedback was embedded in the causal scenario and information about the outcome replaced by a "?" (Experiment 3). A factorial experiment manipulating cover story and feedback revealed that the regression-to-baseline effect was primarily driven by presentation of the "?" feedback (Experiment 4). We conclude that the procedure of testing without feedback, used widely in studies of human cognition, is an appropriate way of assessing learning, as long as the missing data are attributed to the experimenter and the absence of feedback is not highlighted in a way that induces uncertainty.
\end{abstract}




\section{Nonreactive testing: Evaluating the effect of withholding feedback in predictive learning}

Studies of human associative learning phenomena often require separation of training and testing. For example, in a predictive learning task such as the classic "allergist" task (Wasserman, 1990), participants must learn what foods (cues) cause allergic reactions (outcomes) in a fictitious patient. During training, participants are presented with various foods and make predictions about the expected outcome (e.g., allergic reaction or no allergic reaction). Feedback about the actual outcome is then provided to help participants learn. In the case of conditioning procedures, feedback consists of presentations of biologically-salient unconditioned stimuli (US; e.g., electric shocks, also termed "reinforcers") which are paired with conditioned stimuli (CS; e.g., tones). A core principle in associative models is that learning is a function of prediction error, the discrepancy between what is expected and what occurs (e.g., Rescorla \& Wagner, 1972). Pairings of cues and outcomes (or CSs and USs) increase the strength of associations between them (acquisition), while presentation of cues in the absence of outcomes weakens those associations (extinction). This strengthening and weakening of associative links is thought to underlie how humans learn about causal/predictive relationships between cues and outcomes (Gluck \& Bower, 1988; Shanks \& Dickinson, 1987; Le Pelley, Griffiths, \& Beesley, 2017).

In order to prevent further learning (strengthening of associations) from occurring during testing, it is common in conditioning studies to test in the absence of any outcomes (i.e., testing under extinction). This would mean presenting the to-betested CSs with no USs and observing responding. One issue with this procedure is that extinction may have differential effects on the test stimuli. Associative models (e.g., Rescorla \& Wagner, 1972) predict that the amount of extinction will increase over test trials, and that cues that start with higher associative strength will have more negative prediction error and therefore undergo more extinction. Further, there is evidence showing that the rate of extinction depends on the training history of the cue (e.g., partially reinforced cues extinguish slower than continuously reinforced cues, e.g., Humphreys, 1939). Some studies have attempted to remedy this problem of testing under extinction by administering intermittent reinforcement to previously reinforced cues (CS+) at test (e.g., Dunsmoor \& LaBar, 2013). However, this procedure arguably trains a new discrimination: that the $\mathrm{CS}+$ is reinforced and other stimuli are not (Honig \& Urcuioli, 1981). This is particularly problematic when 
assessing generalization as rather than intermittent reinforcement slowing the rate of extinction, rapid decreases in responding might instead occur to all stimuli except the $\mathrm{CS}+$ as participants learn the new discrimination. In sum, both options (presenting or not presenting the outcome) can fail to prevent further learning from occurring at test, making the experimental separation of learning and testing difficult.

One popular solution to this problem in the field of predictive and causal learning is to withhold feedback about the outcome during the test phase, and furthermore to inform participants to expect feedback to be withheld. On such trials, cues are presented and ratings are collected, but they are not followed by either the presence or absence of the outcome; instead no information on the status of the outcome is provided. The underlying assumption is that, if participants do not know whether the outcome would have occurred, prediction error cannot be determined and thus learning at test will be prevented. In this way, learning acquired during training can be assessed without contamination of the later test trials.

This no-feedback testing procedure is ubiquitous in predictive learning experiments. In the simplest case, participants are simply informed that they will not receive feedback for the upcoming test phase (e.g., Lee \& Lovibond, 2021; Lee et al., 2019; Uengoer et al., 2020), or are forewarned that this will occur for the final series of trials (e.g., Shanks \& Darby, 1998; Williams et al., 1994). In fear-conditioning studies, the experimenter can unplug the shock electrodes to show participants that shocks are not possible prior to the test phase; participants are then asked to make hypothetical expectancy ratings of shock (Lee et al., 2018, 2019; Wong \& Lovibond, 2017). Another approach is to intersperse test ("probe") trials, on which no feedback is provided, alongside trials featuring other training cues on which feedback $i s$ provided (Wills et al., 2007, 2014; George \& Kruschke, 2012; Eatherington \& Haselgrove, 2020). Aside from testing purposes, the no-feedback procedure is also used when researchers want to train within-compound associations (e.g., Graham et al., 2011) or simply to expose participants to stimuli during training (e.g., Le Pelley \& McLaren, 2001, 2004). In sum, the no-feedback procedure is used when researchers want to prevent participants learning about cue-outcome relationships.

No-feedback testing can be implemented in a variety of ways. Some studies present alternative feedback in place of outcome feedback. For example, participants may be told that outcome data for particular trials have been "lost" (e.g., Le Pelley \& McLaren, 2001, 2004), that "no information is available" (e.g., Collins \& Shanks, 
2006; George \& Kruschke, 2012), or they may be presented with question marks “???” (e.g., Luque et al., 2017; Wills et al., 2007, 2014). In contrast, other studies do not present any alternative feedback and participants simply progress to the next trial after making their prediction response (e.g., Lee \& Lovibond, 2021; Lee et al., 2019; Livesey et al., 2019; Lee \& Livesey, 2012). Studies also vary in the degree to which the missing-outcome feedback is integrated into the cover story. For example, Le Pelley and McLaren $(2001,2004)$ used an allergist task and instructed participants that allergy results for some of the meals had been lost as the clinic did not always run smoothly. Luque et al. (2017) used a task where participants had to trade cookies with aliens in return for diamond rewards, and participants were told that information about the value of the diamonds would sometimes be unavailable due to solar interference. Alternatively, participants may be told that the missing outcome feedback is part of the experiment (e.g., Shanks et al., 1998). Most commonly, participants are given no additional information and simply told to expect feedback to be withheld in upcoming trials (e.g., Lee \& Lovibond, 2021; Williams et al., 1994).

Despite widespread use of the no-feedback procedure in predictive learning experiments, to date, there has been no attempt to test whether withholding feedback on test trials is effective in preventing learning. Establishing the effectiveness of this procedure is critical if we wish to compare associative learning phenomena in humans and non-human animals, or to test whether predictions from associative theories are borne out in predictive learning scenarios (Dickinson et al., 1984). We tested this proposition using variants of the no-feedback testing procedure in an allergist task. Experiment 1 used the common method of withholding feedback for an entire test phase, while Experiments 2-4 tested the effect of withholding feedback for specific trials.

\section{Experiment 1}

\section{Method}

\section{Participants}

All experiments were approved by the UNSW Human Research Ethics Advisory Panel (Psychology), and were conducted online. Experiments 1-3 used a sample of first-year Psychology students from the University of New South Wales, who participated in exchange for course credit. In all experiments reported here, we aimed for a target sample size of at least 34 participants per group after exclusions of non-learners, which would provide power $>.8$ to detect a medium-sized effect $(d=$ 
0.5 ) in a paired t-test (since the critical contrasts were within-subjects). Moving beyond considerations of power for frequentist statistics, where null results were potentially informative we used Bayesian analyses to assess the strength of support for the null hypothesis based on our achieved sample sizes. We tested a total of 82 participants in Experiment 1 (age $M=19.3, S D=2.94,61$ female, 21 male).

Participants were randomly allocated to either the No-Feedback $(n=43)$ or Extinction $(n=39)$ Groups.

\section{Materials}

Experiments were coded in JavaScript using the jsPsych library (de Leeuw, 2015), hosted using JATOS (Lange, Kühn, \& Filevich, 2015) and run on participants' own web browsers. The food pictures used as cues were $300 \times 300$ pixel image files with a colored image and verbal label underneath. The outcomes were $609 \times 380$ pixel image files, either with the message "Allergic Reaction!" accompanied by a sad emoticon or the words "No allergic reaction". The experiment code and images can be found at https://osf.io/dvn9g/.

\section{Design and Procedure}

Participants played through a fictitious scenario in which their task was to diagnose food allergies occurring in $\mathrm{Mr}$ X. They were told that $\mathrm{Mr}$ X wrote down foods he was eating each day in a diary, along with whether he suffered an allergic reaction or not. On each trial, participants would view the contents of one meal, and make a prediction about the outcome (predictive rating) using a visual analogue scale. The trial types presented in each phase of the task are shown in Table 1; letters represent different food cues, while "+" and "-." indicate that the cue was followed by explicit feedback (outcome or no outcome respectively). 
Table 1.

Design of the four experiments.

\begin{tabular}{ccccc}
\hline Experiment & Phase 1 & Phase 2 & Test & $\begin{array}{c}\text { Causal } \\
\text { Ratings }\end{array}$ \\
\hline 1 & & A B & & \\
(No-Feedback Group) & A+ B- & & A B & A B \\
\cline { 3 - 4 } (Extinction Group) & C+ D- & A- B- & C D & C D \\
\cline { 5 - 5 } $2,3, \& 4$ & A+ B- & A B & A B & A B \\
& C+ D- & & C D & C D \\
& E+ F- & E- F- & E F & E F \\
& G+ & G & G & G \\
\hline
\end{tabular}

Note: Letters represent different cues (foods), + represents the outcome (allergic reaction), - represents the absence of the outcome (no allergic reaction). Cues in bold were presented with no feedback. For all experiments, the key (within-subjects) comparisons were between the no-feedback cues (A and B, bolded) and the control cues $(\mathrm{C}$ and $\mathrm{D})$.

Participants in the No-Feedback Group were initially trained with a pair of target cues (A and B), one of which predicted the outcome $(\mathrm{A}+$, i.e., was reinforced) and the other predicted no outcome (B-, i.e., was non-reinforced). These target cues were then repeatedly presented in the absence of feedback. To test our main hypothesis, we compared responding to these target cues with responding to control cues $(\mathrm{C}$ and $\mathrm{D})$ that were equated in terms of reinforcement history but had not been presented without feedback. If participants learn something about the target cues in the absence of outcome feedback, we would expect a difference between the target and control cues on test. If however, the no-feedback procedure is effective in preventing learning, then we expect to see no difference between target and control cues. While our key manipulation in Experiment 1 was within-subjects, we also included an additional group who received the same presentations of the target cues but with explicit "no outcome" feedback (i.e., extinction training). This group allowed us to examine to what degree extinction occurred in our task, and to provide an extreme alternative to compare any effects of the no-feedback manipulation. Thus, Experiment 1 included a between-subjects manipulation whereby participants either received presentations of the target cues with outcome feedback withheld (NoFeedback Group) or explicit "no outcome” feedback (Extinction Group).

In the first (training) phase, participants in both groups learned about two cues that predicted the outcome (A and $\mathrm{C}$ ) and two cues that predicted no outcome (B and D). Each cue was presented four times in phase 1, in random order. Each trial 
consisted of presentation of a single food, and 500ms later, the prompt "Please rate the likelihood that $\mathrm{Mr} \mathrm{X}$ will show an allergic reaction after eating this meal" appeared along with the rating scale, which was a slider ranging from "Definitely no allergic reaction" (scored as 0 ) to "Definitely allergic reaction" (scored as 100), with 1-point increments. The slider's pointer appeared at the midpoint of the scale by default; participants were required to click on the scale and/or move the pointer, at which point a "Continue" button appeared. Participants had unlimited time to adjust their rating, before clicking the "Continue" button to proceed. In the training phases, feedback about the outcome (either "allergic reaction" or "no allergic reaction") then replaced the slider scale and was presented for $2 \mathrm{~s}$ alongside the picture of the food, followed by a blank 1s inter-trial-interval (ITI).

\begin{tabular}{|l|l|}
\hline $\begin{array}{c}\text { apple } \\
\text { Please rate the likelihood that Mr X will show } \\
\text { an allergic reaction after eating this meal }\end{array}$ \\
$\begin{array}{c}\text { Definitely } \\
\text { NO Allergic Reaction }\end{array}$ \\
Allergic Reaction
\end{tabular}

Figure 1. Schematic illustration of each training trial. Participants made predictive ratings about the allergic reaction outcome for each food cue on a visual analogue scale (left panel), and could receive feedback about whether an outcome occurred (right panel).

In phase 2, we manipulated whether participants were given feedback about the outcome for the target cues (A and B). Participants in the Extinction Group continued to receive feedback on the actual outcome that occurred on each trial where cues $\mathrm{A}$ and $\mathrm{B}$ were presented and paired with no outcome. Note that this meant that $\mathrm{A}$ was explicitly extinguished, while B continued to be non-reinforced. In contrast, participants in the No-Feedback Group were given instructions prior to phase 2 that for the next phase, they would no longer be receiving feedback, and to continue making predictions as before. Thus, the No-Feedback Group saw cues A and B for the same number of trials as the Extinction Group, but were not given explicit feedback 
about the absence of the outcome. Note that due to this large difference in procedure between the two groups, we expected a large difference in how participants in those different groups responded to the target cues (A and B) at test. However, the comparisons that were most relevant to our aims (testing whether no-feedback presentations had any effect on predictive or causal judgments), were the subtler comparisons between A vs. C and B vs. D in the No-Feedback group. Each cue was presented four times in phase 2.

After phase 2, participants in both groups were then administered the same test phase in which all cues (A-D) were presented twice each. Participants in the Extinction Group were told prior to the start of the test phase that feedback would be withheld for the following phase (just as the No-Feedback Group were told prior to the second phase). For the No-Feedback Group, the transition to the final test phase was seamless since they had already been given the relevant instruction that feedback would be withheld. Note that although the test phase did not differ from phase 2 for the no-feedback cues in this and subsequent experiments, the inclusion of a test phase was critical to allow us to compare the no-feedback cues to the control cues, since these control cues were not presented in phase 2.

Participants then completed a causal rating test in which for each cue (A-D, presented once) participants were asked "Please rate to what extent this food tended to cause an allergic reaction". Participants entered their causal rating using a slider which ranged from "NO effect" (scored as 0 ) to "Strongly CAUSED allergic reaction" (scored as 100). Note that although there are similarities in the wording of the questions in the predictive and causal ratings, it has been argued that phrasing the question in terms of conditional probability of the outcome might index different aspects of learning compared to asking questions about causal status (Matute et al., 1996; Collins \& Shanks, 2002). Trial order within each phase (training, test, causal ratings) was randomized in blocks consisting of one presentation of each trial type.

Finally, participants completed some questionnaire items that aimed to encourage comparison between target and control cues and elicit explanations for why these cues might differ in causal status. Note that these questions were included for exploratory purposes and will not be discussed in detail here (see Supplemental Materials for full details and results).

\section{Results}

\section{Data Analysis}


Data were analyzed in R (R Core Team, 2019) using the "afex" (Singmann et al., 2019), and "emmeans" (Lenth, 2019) packages, and the "BayesFactor" package (Morey \& Rouder, 2018) with the default prior. For all experiments, the key (withinsubjects) comparisons were between the no-feedback cues (A and B, bolded in Table 1) and the control cues (C and D). We performed these planned comparisons for the test phase, and also for the causal ratings, using one-way ANOVAs (analysis of variance) and Bayesian t-tests. Factorial ANOVAs were run for analyses involving group effects (Experiments 1 and 4), and linear trend contrasts were computed to examine the effect of withholding feedback across trials.

\section{Exclusion Criteria}

Following previous predictive learning studies from our lab (Lee \& Lovibond, 2021), participants were excluded if in the last 2 trials for each stimulus in the first training phase, their mean rating for $\mathrm{A}$ and $\mathrm{C}$ (the reinforced cues) was below 75 or if their mean rating for $\mathrm{B}$ and $\mathrm{D}$ (the nonreinforced cues) was above 25 . This resulted in 9 participants being excluded, leaving 35 in the Extinction Group and 38 in the NoFeedback Group ${ }^{1}$.

\section{Training}

Figure 2a shows mean predictive ratings across training phases 1 and 2, and the test phase, for both groups. It is clear that participants in both groups acquired the appropriate cue-outcome contingencies during phase 1, coming to give high predictive ratings to cues that predicted the outcome (A and $\mathrm{C}$ ) and low predictive ratings to cues that predicted no outcome (B and D). Unsurprisingly, both groups showed similar performance during this acquisition phase as their treatment was identical up to this point. Given that patterns of responding in the acquisition phase were so clear, for the sake of brevity we do not report inferential analyses of these data here or in subsequent experiments.

We assessed learning across phase 2 -in which the no-feedback manipulation was implemented - using linear trend analysis. In the Extinction Group, pairing A with no outcome resulted in a linear decrease in ratings across the 4 trials (i.e., extinction), $F(1,102)=88.0, p<.001, \eta_{p}{ }^{2}=.46$, MSE $=947.7$, while for $\mathrm{B}$, ratings remained low and did not show a significant linear trend, $F<1$. In the No-Feedback

\footnotetext{
${ }^{1}$ Application of this training criterion resulted in changes to the statistical significance of a single result in Experiment 3, noted below. It did not change the pattern of statistical significance or support for the alternative over the null (or vice versa) for any of the other results.
} 
Group, ratings did not seem to change across phase 2 for either the previously reinforced (A) or previously non-reinforced (B) cues, supported by non-significant linear trends for both cues, $F \mathbf{s}<1$.

A

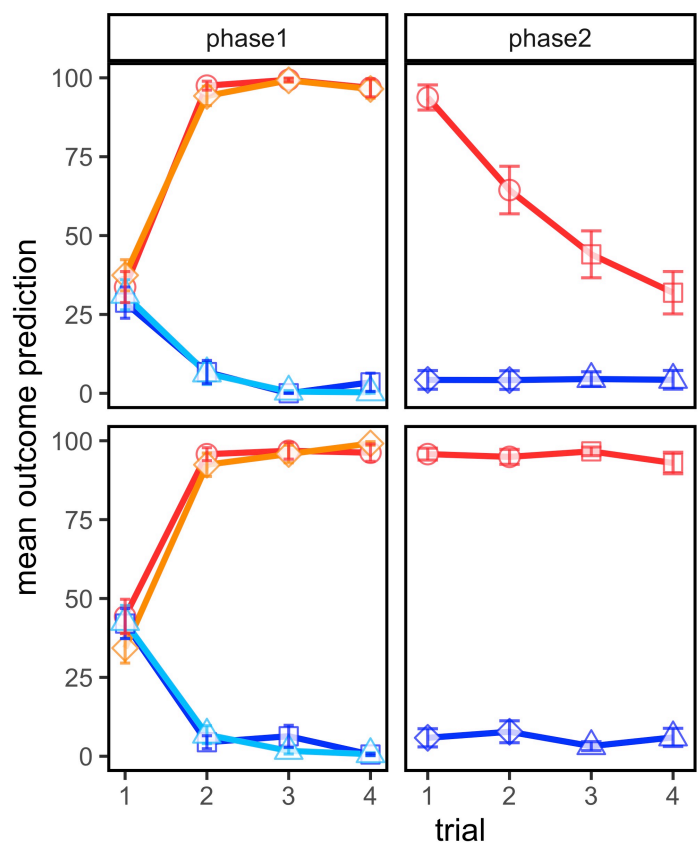

B

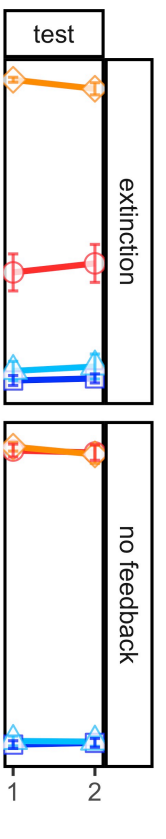

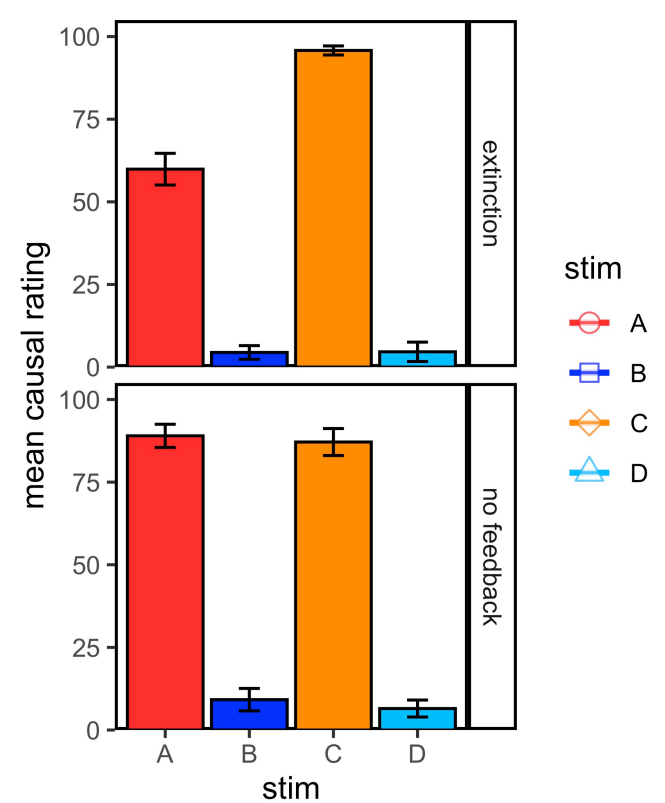

Figure 2. A) Mean predictive ratings across training phases 1 and 2, and the test phase, and B) mean causal ratings for the Extinction (top row) and No-Feedback (bottom row) Groups, in Experiment 1. Error bars indicate standard error of the mean.

\section{Outcome Prediction Test}

Data from the test phase were analyzed by first averaging across the two test trials for each cue and conducting a series of between- and within-subjects one-way ANOVAs and Bayesian t-tests. Not surprisingly, the Extinction group gave lower ratings than the No-Feedback Group for cue A, $F(1,71)=97.3, p<.001, \eta_{p}{ }^{2}=.58$, $\mathrm{MSE}=604.5, \mathrm{BF}_{10}>10^{11}$, but not for cue $\mathrm{B}, F<1, \mathrm{BF}_{10}=0.25$. The results of most interest to our primary hypothesis came from a comparison of the cues that appeared without feedback in phase 2 (A and B) with the control cues that did not appear in phase $2(\mathrm{C}$ and $\mathrm{D})$ in the No-Feedback Group (Figure 2a). This contrast revealed no significant difference in predictive ratings for A versus $\mathrm{C}, F<1, \mathrm{BF}_{10}=0.18$, or in ratings for $\mathrm{B}$ versus $\mathrm{D}, F<1, \mathrm{BF}_{10}=0.25$; in both cases the Bayes Factor suggests moderate evidence in support of the null hypothesis (no difference) over the alternative (Jeffreys, 1939). 
Figure $2 \mathrm{~b}$ shows the mean causal ratings for each cue. The pattern was similar to that of the outcome prediction test. The Extinction Group gave significantly lower causal ratings to cue A than did the No-Feedback Group, $F(1,71)=24.5, p<.001, \eta_{p}{ }^{2}$ $=.26, \mathrm{MSE}=629.3, \mathrm{BF}_{10}=3330.7$, but causal ratings to $\mathrm{B}$ did not differ significantly between groups, $F(1,71)=1.37, p=.246, \eta_{p}{ }^{2}=.02, \mathrm{BF}_{10}=0.43$. Critically, in the NoFeedback Group, causal ratings did not differ significantly when comparing A and C, $F<1, \mathrm{BF}_{10}=0.19$, nor between $\mathrm{B}$ and $\mathrm{D}, F(1,37)=1.84, p=.184, \eta_{p}{ }^{2}=.05, \mathrm{BF}_{10}=$ 0.41 .

In summary, Experiment 1 showed that withholding feedback during an entire phase did not affect subsequent test or causal ratings relative to control cues that were not presented during the same test phase. By contrast, there were clear effects of extinction with decreasing ratings to cue A over trials of phase 2 in the Extinction group, and clear group differences for cue $\mathrm{A}$ in both the predictive ratings and causal ratings tests.

\section{Experiments 2 and 3}

Experiments 2 and 3 used a within-subjects design to examine the effect of withholding outcome feedback for specific trials. This procedure can be likened to the practice of using "probe" trials interspersed among other trials in which cues are presented with their respective outcomes during training (Wills et al., 2007, 2014; George \& Kruschke, 2012; Eatherington \& Haselgrove, 2020). However, unlike typical practice with probe trials, we withheld feedback for specific cues in a continuous block of training trials (similar to a test phase). Our rationale was that if withholding feedback had no effect here, then it would presumably also have no effect if the no-feedback trials were interspersed as probe trials. In other words, this procedure, although slightly unusual, constitutes a strong test of the effect of withholding feedback and makes a null result more informative.

Experiments 2 and 3 were identical except for two differences in the way the missing feedback was presented. Experiment 2 was designed so that participants would attribute the missing feedback to the experimenter, while Experiment 3 was designed so that participants might have reasons to suspect that the missing feedback was being systematically censored. Specifically, Experiment 2 implemented the nofeedback procedure in a similar way to Experiment 1, by informing participants that for some trials they would not be receiving feedback and simply omitting the outcome feedback for those trials. In contrast, Experiment 3 embedded the no-feedback 
instruction in the cover story: participants were told that $\mathrm{Mr} \mathrm{X}$ sometimes forgot to write down whether he suffered an allergic reaction or not, and that on those days, participants would see a "?" in place of the outcome feedback (similar to Luque et al., 2017; Wills et al., 2007, 2014).

Method

Participants

A total of $66(M$ age $=19.4, S D$ age $=2.33,48$ female, 17 male, 1 other $)$ and $60(M$ age $=20.0, S D$ age $=2.08,40$ female, 18 male, 2 other $)$ University of New South Wales students participated in Experiments 2 and 3 respectively, for course credit.

\section{Design and Procedure}

The procedure was identical to Experiment 1 except for the following changes. Compared to Experiment 1, there were more cues in Phase 1 (4 reinforced and 3 non-reinforced cues) and the training phases were slightly longer, with 6 repetitions of each trial type in each phase. There was also no between-group manipulation in Experiments 2 and 3; all comparisons were within-subjects. The main difference however, was that rather than withholding feedback for all cues in a test phase, in these experiments, feedback was withheld for specific cues—but not others - during a second phase of training.

In the first phase, participants were presented with 4 cues that predicted the outcome (A, C, E, G) and 3 cues that predicted no outcome (B, D, F, see Table 1). In the second phase, the target cues A and B were presented with no feedback, cue E was extinguished, and cue F continued to be paired with the absence of the outcome, while the control cues $\mathrm{C}$ and $\mathrm{D}$ were not presented. An additional cue, G, was paired with the outcome in both phases 1 and 2; this cue was included to discourage participants from forming the belief that allergic reaction (in general) was no longer possible in phase 2 (which might otherwise have been the case, given that all other cues were either non-reinforced or had feedback withheld in phase 2).

Experiments 2 and 3 differed in two procedural details. The first difference was in the instructions given to explain the absence of feedback. In Experiment 2, participants were told "Note that on some trials you will not receive feedback about whether an allergic reaction occurred.". This instruction is similar to the pre-test instructions used in Experiment 1, and was intended to be neutral with regards to the reason for the missing feedback. In contrast, participants in Experiment 3 were told 
"Note that on some days, Mr X forgot to write down whether he suffered an allergic reaction or not. On those days, you will see a "?" and will not be given any feedback.". This instruction is similar to previous studies in providing a reason for the missing data in the context of the cover story (e.g., Le Pelley \& McLaren, 2001; Luque et al., 2017). The second difference was how the missing feedback trials were presented. After participants made their predictive rating, the target cues A and B were presented on an otherwise blank screen in Experiment 2, but with a "?" symbol in Experiment 3. The cues, along with this missing feedback, appeared for the same amount of time (2s) as the outcome feedback was shown for the other cues.

As in Experiment 1, participants were then assessed in a test phase in which they were informed that all feedback would be withheld but that they should continue to make outcome predictions as before. This outcome prediction test was followed by a causal ratings test, again as in Experiment 1 . All cues (A-G) were assessed in both test phases, with two presentations of each cue in the outcome prediction test and one presentation of each cue in the causal ratings test. Questionnaire items were also administered at the end of the experiment (see Supplemental Materials).

\section{Results}

After applying the same exclusion criteria as in Experiment 1 there were 58 participants remaining in Experiment 2, and 52 in Experiment 3.

\section{Training}

Figures $3 \mathrm{a}$ and $3 \mathrm{c}$ show that the cue-outcome contingencies were learned rapidly in the acquisition phase (phase 1) of Experiments 2 and 3. Analysis of data from phase 2 used linear trend analysis to examine whether predictive ratings for the no-feedback cues (A and B) changed over training trials. In Experiment 2, the linear trend approached but did not reach significance for cue A, $F(1,255)=3.45, p=.064$, $\eta_{p}{ }^{2}=.01$, and was non-significant for cue $\mathrm{B}, F<1$ (Figure 2a). By contrast, in Experiment 3 both cues A, $F(1,255)=40.0, p<.001, \eta_{p}{ }^{2}=.14$, MSE $=537.8$, and B, $F(1,255)=40.2, p<.001, \eta_{p}{ }^{2}=.14, \mathrm{MSE}=267.3$, showed a significant linear trend, with ratings decreasing for $\mathrm{A}$ and increasing for $\mathrm{B}$ over trials (Figure $3 \mathrm{c}$ ). 
A
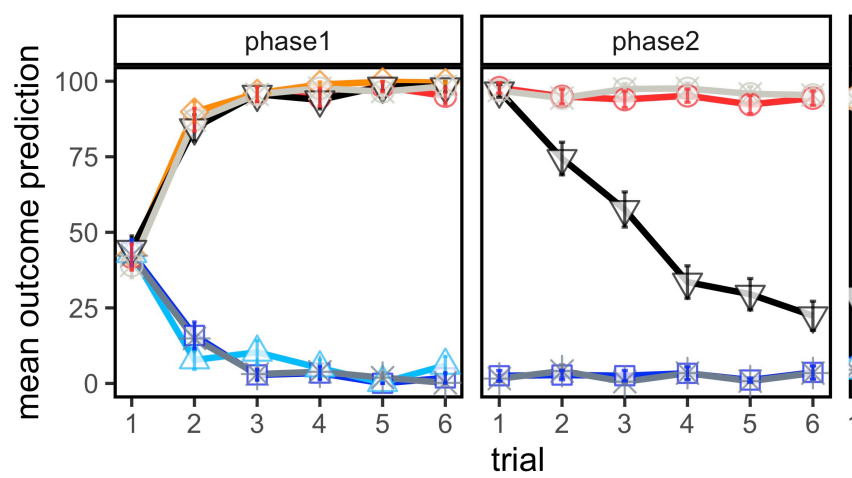

C

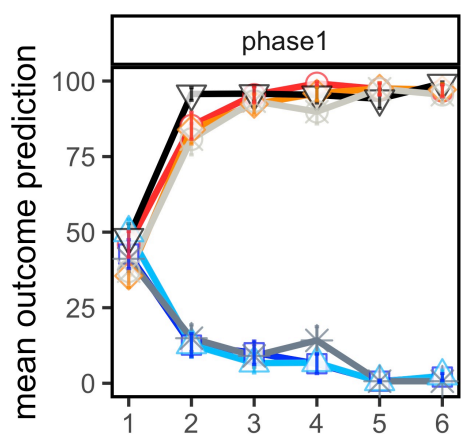

B

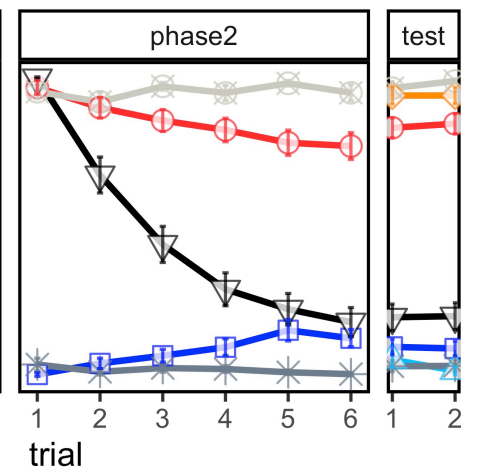

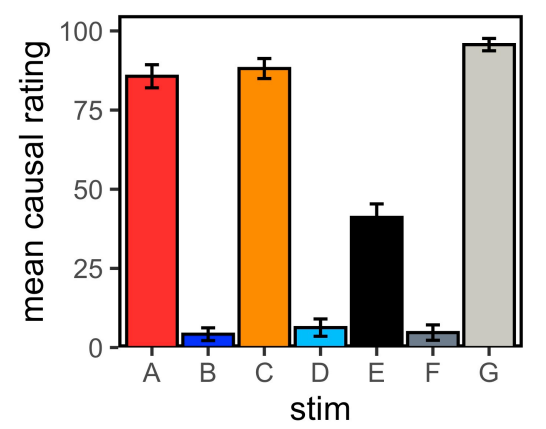

$\mathrm{D}$
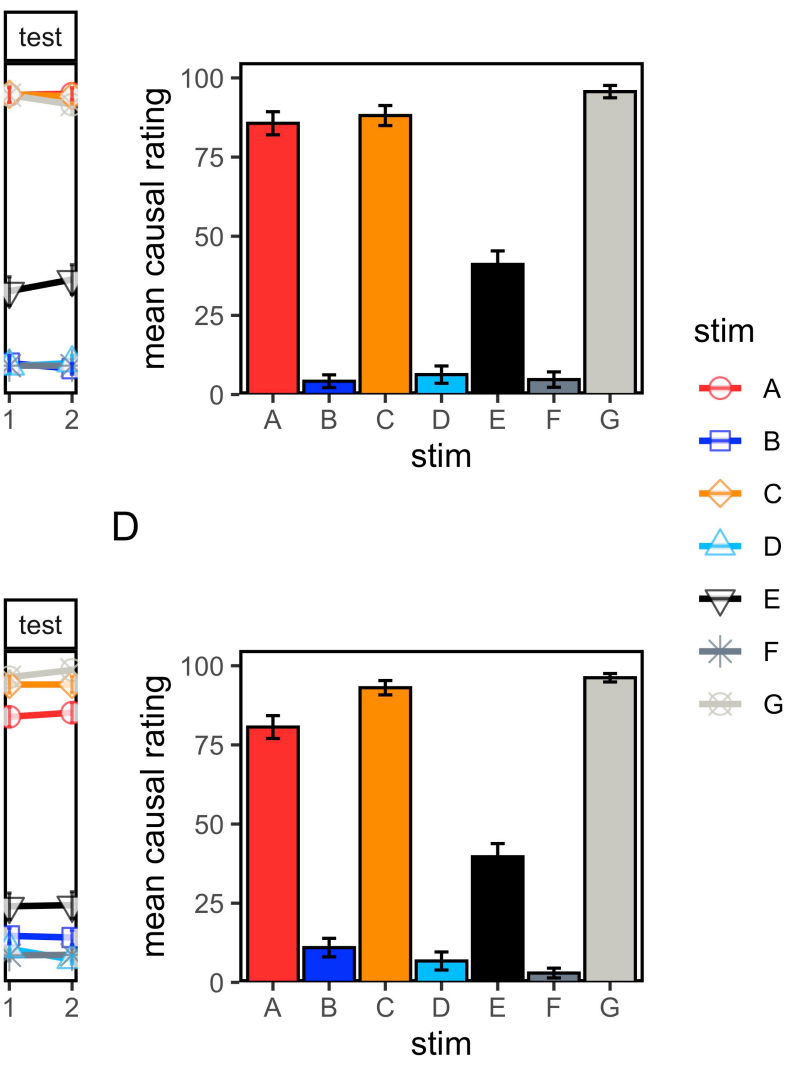

Figure 3. A) Mean predictive ratings across the training (phase 1 and phase 2) and test phases in Experiment 2. B) Mean causal ratings in Experiment 2. C) Mean predictive ratings across training and test phases in Experiment 3. D) Mean causal ratings in Experiment 3. Error bars indicate standard error of the mean.

\section{Outcome Prediction Test}

Data from the test phase were consistent with the idea that ratings for A and B had changed little over the course of the second training phase in Experiment 2 (Figure 3a), but had changed more substantially in Experiment 3 (Figure 3c). There was no significant difference between predictive ratings for cues $\mathrm{A}$ and $\mathrm{C}$, nor between cues B and D in Experiment 2, $F \mathrm{~s}<1, \mathrm{BFs}_{10}<0.146$, with the Bayes Factors providing moderate support for the null hypothesis. In contrast, cue A was rated significantly lower than cue $\mathrm{C}, F(1,51)=7.54, p=.008, \eta_{p}{ }^{2}=.13, \mathrm{MSE}=345.0, \mathrm{BF}_{10}$ $=4.33$, and cue $\mathrm{B}$ was rated significantly higher than cue $\mathrm{D}, F(1,51)=4.52, p=.038$, $\eta_{p}{ }^{2}=.08, \mathrm{MSE}=193.5, \mathrm{BF}_{10}=1.19$, in Experiment $3^{2}$.

\footnotetext{
${ }^{2}$ Note that this comparison was not significant and the BF indicated support for the null when including the participants who failed the training criterion.
} 


\section{Causal Ratings Test}

The pattern of causal ratings was similar to that for the outcome prediction test. In Experiment 2 (Figure 3c), there were no significant differences between causal ratings for cues $\mathrm{A}$ and $\mathrm{C}$, nor between cues $\mathrm{B}$ and $\mathrm{D}, F_{\mathrm{S}}<1.1, \mathrm{BFs}_{10}<0.243$, with the Bayes Factors providing moderate support for the null hypothesis. In Experiment 3 (Figure 3d), Cue A was rated significantly lower than Cue C, $F(1,51)=13.6, p<$ $.001, \eta_{p}{ }^{2}=.21, \mathrm{MSE}=295.1, \mathrm{BF}_{10}=48.1$, but there was no significant difference between cues $\mathrm{B}$ and $\mathrm{D}, F(1,51)=1.15, p=.290, \eta_{p}{ }^{2}=.02, \mathrm{BF}_{10}=0.259$.

\section{Discussion}

Despite only minor procedural differences between Experiments 2 and 3, there were marked differences in how participants responded to the no-feedback cues (A and B). When participants were told that $\mathrm{Mr} \mathrm{X}$ sometimes forgot to write down his allergic reactions (Experiment 3), participants showed a regression-to-baseline effect, with predictions for both the previously reinforced cue A and the previously nonreinforced cue B gravitating towards the midpoint of the scale. In contrast, when participants were simply told to expect no feedback (Experiment 2), there was no such regression effect. In both experiments, the trends observed during training carried over to the outcome prediction test and causal ratings, although the regressionto-baseline effect at test in Experiment 3 appeared to be more pronounced for cue A than for B.

The results of Experiment 3 may be explained by the fact that the no-feedback instructions were integrated into the cover story and attributed to $\mathrm{Mr} \mathrm{X}$; that is, participants were told that feedback was omitted when $\mathrm{Mr}$ X forgot to write down whether he suffered a reaction or not. This may have encouraged participants to think about reasons for the missing feedback. For instance, some participants reported thinking that $\mathrm{Mr} \mathrm{X}$ was more likely to forget to write down his reactions when he experienced no allergic reaction (see Supplemental Materials). Hence, the missing data could have been interpreted as informative of which outcome had actually occurred. If the interpretation relating to the identity of the censored outcome varied across participants, it could explain the overall regression towards the midpoint of the scale that was observed in Experiment 3. By contrast, in Experiment 2 no reason was provided for the missing feedback - participants were simply told that feedback would sometimes be omitted — which may have discouraged speculation about whether there was any deeper meaning regarding when feedback was omitted. 
Alternatively, it is possible that Experiment 3's approach of presenting a "?" symbol in place of outcome feedback - as opposed to a blank screen in Experiment 2 - was responsible for the systematic changes in responding despite no information being presented about the allergy outcomes. This procedure was intended to complement the cover story by providing a clear visual cue to indicate the meals in which $\mathrm{Mr} \mathrm{X}$ forgot to write down his reactions, but may have had an effect on participants' interpretation of the missing-feedback trials in its own right. To test whether the cover story or the presentation of the alternative "?" feedback was responsible for the regression-to-baseline effect observed in Experiment 3, we ran a further experiment to isolate the effect of these two variables.

\section{Experiment 4}

The aim of Experiment 4 was to determine which of the two key procedural differences between Experiments 2 and 3 led to the regression-to-baseline effect observed for the no-feedback cues. Experiment 4 was a 2x2 design, with cover story (neutral vs. MrX), and missing-feedback indicator (blank vs. "?") as between-subjects factors. Consequently, the neutral-blank group was a replication of Experiment 2, and the MrX-? group was a replication of Experiment 3.

Method

\section{Participants}

A total of $239(M$ age $=26.9, S D$ age $=7.9,90$ female, 141 male, 6 other, 2 who did not answer) workers were recruited for this experiment through the Prolific (prolific.co) online platform. Participants were paid GBP£2 for a 15 minute study. A total of 59 participants were allocated to the MrX-? group, 64 to the MrX-blank group, 56 to the neutral-? group, and 60 to the neutral-blank group.

\section{Design and Procedure}

The procedure was identical to Experiments 2 and 3 except that participants were randomly allocated to either the neutral cover story of Experiment 2 (in which no reason for missing feedback was provided), or the MrX cover story of Experiment 3 (in which feedback was missing because $\mathrm{Mr} \mathrm{X}$ sometimes forgot to write down his allergic reactions). They were also randomly allocated to receive either a blank screen (as in Experiment 2) or a "?" (as in Experiment 3) as the indicator on the no-feedback trials. In both cases, the feedback period was the same as the previous experiments (2s). The critical section of the instructions given to each group prior to training was as follows (with bold text as in the original): 
- MrX-? group: "Note that Mr X sometimes forgets to write down whether he suffered an allergic reaction or not. On those days, you will see a "?" and will NOT be given feedback about whether or not an allergic reaction occurred."

- MrX-blank group: "Note that Mr X sometimes forgets to write down whether he suffered an allergic reaction or not."

- Neutral-? group: "Note that on some trials you will not receive feedback about whether an allergic reaction occurred. On those trials, you will see a "?" and will NOT be given feedback about whether or not an allergic reaction occurred."

- Neutral-blank group: "Note that on some trials you will not receive feedback about whether an allergic reaction occurred."

Questionnaire items were again administered at the end of the experiment. Of most importance was a forced-choice question probing participants' explanations of the missing feedback (see Supplemental Materials). We included two options regarding the assumed magnitude of the censored outcome (the allergic reaction did not happen or may have been very small; the allergic reaction may have been very large), an agnostic or neutral option (no reason/I didn't think too much about this), as well as a combined uncertain/suspicious option (I didn't know but it confused me/made me suspicious).

\section{Results}

After applying the same exclusion criteria as the previous experiments there were 226 participants remaining: 57 in the MrX-? group, 61 in the MrX-blank group, 51 in the neutral-? group, and 57 in the neutral-blank group.

\section{Training}

Figure 4a shows mean predictive ratings across training phases 1 and 2, and the test phase, for all groups. We assessed learning across phase 2 using linear trend analysis over trials in factorial ANOVAs with cover story and indicator as betweensubjects factors. Separate ANOVAs were run for cues A and B. For cue A, there was a significant overall negative linear trend across trials, $F(1,1110)=133.1, p<.001$, $\eta_{p}{ }^{2}=.11, \mathrm{MSE}=413.7$, a significant main effect of indicator group, $F(1,222)=19.9$, $p<.001, \eta_{p}{ }^{2}=.08, \mathrm{MSE}=1835.1$, and also a significant interaction between indicator group and linear trend, $F(1,1110)=9.88, p=.002, \eta_{p}{ }^{2}=.01$, MSE $=413.7$. The latter interaction reflected the finding that ratings for A showed a greater decrease over the 
course of phase 2 training in the groups with missing feedback indicated by "?" as compared to those who saw a blank screen. The main effect of cover story, and other interactions were all non-significant, $F_{\mathrm{S}}<2.44, p \mathrm{~s}>.119$. The pattern of results was similar for cue $\mathrm{B}$, with an overall significant linear trend across trials, $F(1,1110)=$ $20.8, p<.001, \eta_{p}{ }^{2}=.02, \mathrm{MSE}=304.4$, a significant main effect of indicator group, $F(1,222)=23.7, p<.001, \eta_{p}{ }^{2}=.10, \mathrm{MSE}=1214.0,$, and a significant interaction between indicator group and linear trend $F(1,1110)=11.2, p<.001, \eta_{p}^{2}=.01, \mathrm{MSE}=$ 304.4. Here, the interaction reflected the finding that ratings for B showed a greater increase over the course of phase 2 training in the "?" groups relative to the blank groups. No other main effects or interactions were significant, $F_{\mathrm{S}}<2.03, p \mathrm{~s}>.154$.

A

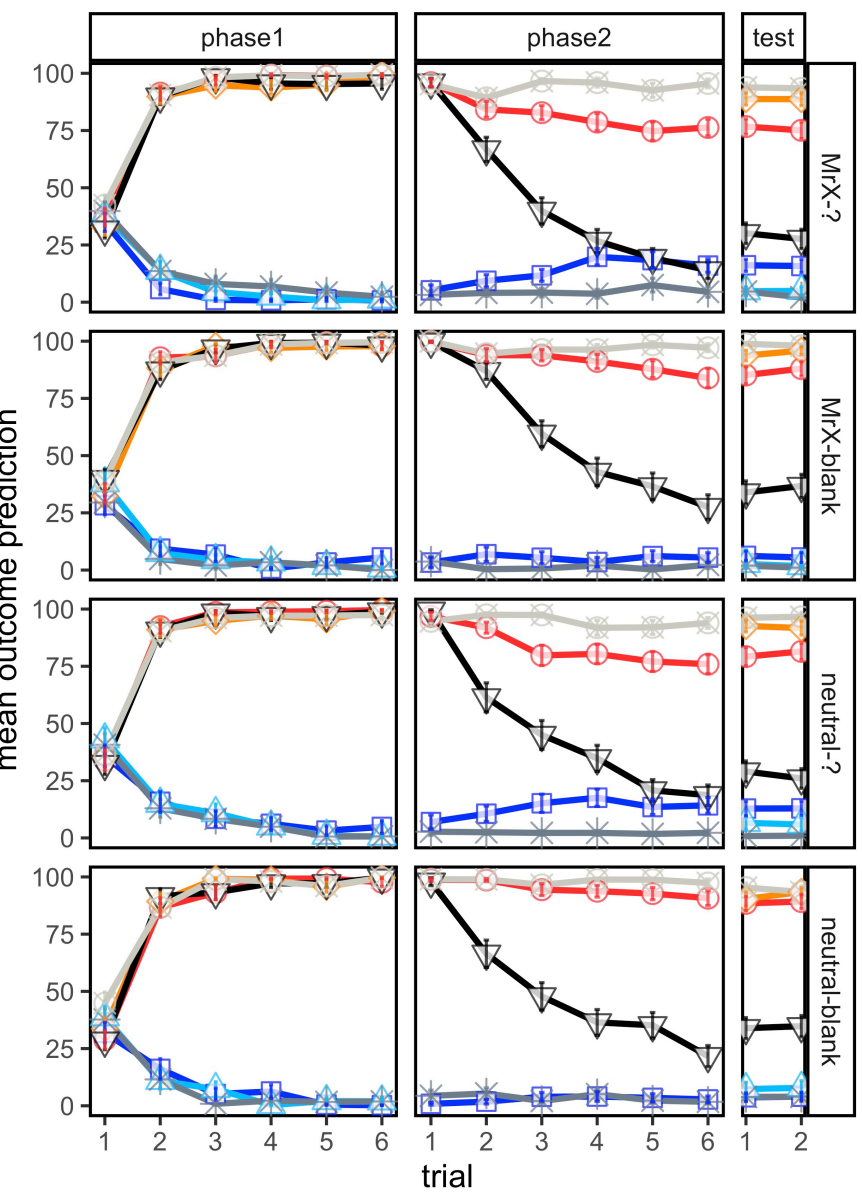

B

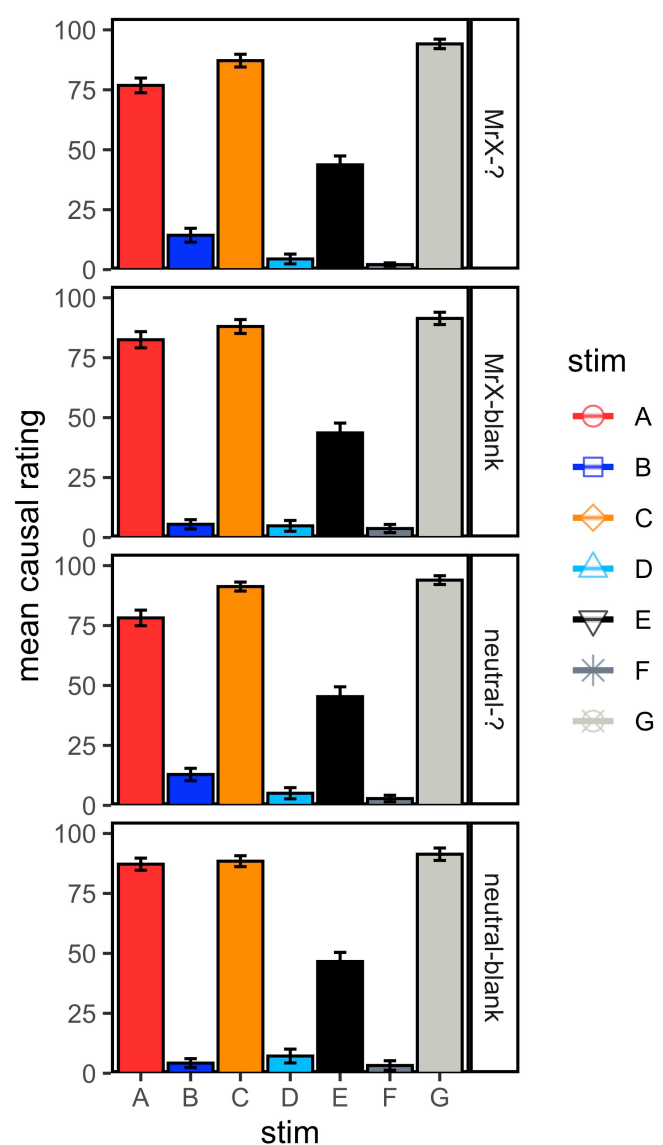

Figure 4. A) Mean predictive ratings across the training (phase 1 and phase 2) and test phases in Experiment 4. B) Mean causal ratings in Experiment 4. Error bars indicate standard error of the mean. Group names are shown on the right of each graph. 


\section{Outcome Prediction Test}

The data from the test phase were first analyzed in an ANOVA with cover story and indicator as the between-subjects factors, and stimulus (no-feedback vs. control) as the within-subjects factor. Separate ANOVAs were run for the reinforced cues (comparing cue A to control cue C) and for the non-reinforced cues (comparing cue B to control cue D).

For the reinforced cues, there was a significant main effect of stimulus, $F(1,222)=28.5, p<.001, \eta_{p}{ }^{2}=.11, \mathrm{MSE}=317.7$, with lower ratings for $\mathrm{A}$ than for the control cue $\mathrm{C}$ overall. There was also a significant main effect of indicator group, $F(1,222)=7.37, p=.007, \eta_{p}{ }^{2}=.03, \mathrm{MSE}=597.3$, and an interaction between stimulus and indicator group, $F(1,222)=3.97, p=.048, \eta_{p}{ }^{2}=.02, \mathrm{MSE}=317.7$, with a greater difference between $\mathrm{A}$ and $\mathrm{C}$ in the "?" groups than the blank groups. All other main effects and interactions were non-significant, $F_{\mathrm{S}}<1$. The pattern of results was similar for the non-reinforced cues, with significantly higher ratings to cue $\mathrm{B}$ overall when compared to the control cue $\mathrm{D}, F(1,222)=10.6, p=.001, \eta_{p}{ }^{2}=.05$, $\mathrm{MSE}=207.7$, a significant main effect of indicator group, $F(1,222)=7.10, p=.008$, $\eta_{p}{ }^{2}=.03, \mathrm{MSE}=415.0$, and a significant interaction between stimulus (B vs. D) and indicator group, $F(1,222)=10.8, p=.001, \eta_{p}{ }^{2}=.05, \mathrm{MSE}=207.7$, with a greater difference between B and D in the "?" groups than the blank groups. However, unlike with the A vs. C comparison, there was also a significant interaction between cover story group and the $\mathrm{B}$ vs. D comparison, $F(1,222)=4.74, p=.030, \eta_{p}{ }^{2}=.02$, $\mathrm{MSE}=$ 207.7, reflecting a greater difference between $\mathrm{B}$ and $\mathrm{D}$ in the MrX groups than the neutral groups. The remaining effects were non-significant, $F \mathbf{s}<1$.

We then performed simple effect comparisons (A vs C, B vs. D) in each of the four groups, using Holm-Bonferroni correction to control for inflation of Type 1 errors (Holm, 1979). For ease of presentation, we report the uncorrected p-values and note where the pattern of significance changed as a result of correction. Participants rated $\mathrm{A}$ significantly lower than $\mathrm{C}$ in the in the $\mathrm{MrX}-$ ? group, $F(1,56)=17.5, p<$ $.001, \eta_{p}{ }^{2}=.24, \mathrm{MSE}=268.6, \mathrm{BF}_{10}=214.5$, and in the Neutral-? group, $F(1,50)=$ $8.98, p=.004, \eta_{p}{ }^{2}=.15, \mathrm{MSE}=396.2, \mathrm{BF}_{10}=7.86$. The same comparison was not significant in the MrX-blank Group after Holm-Bonferroni correction, $F(1,60)=4.57$, $p=.037, \eta_{p}{ }^{2}=.07, \mathrm{BF}_{10}=1.16$, and there was no significant difference in the Neutral-Blank group, $F(1,56)=1.70, p=.197, \eta_{p}{ }^{2}=.03, \mathrm{BF}_{10}=0.323$. 
Participants rated $\mathrm{B}$ significantly higher than $\mathrm{D}$ in the $\mathrm{MrX}-$ ? group, $F(1,56)=$ $13.8, p<.001, \eta_{p}{ }^{2}=.20, \mathrm{MSE}=254.1, \mathrm{BF}_{10}=54.1$, and the MrX-blank group, $F(1,60)=8.96, p=.004, \eta_{p}{ }^{2}=.13, \mathrm{MSE}=45.5, \mathrm{BF}_{10}=7.73$, but no significant difference was found in the neutral-? group, $F(1,50)=3.03, p=.088, \eta_{p}^{2}=.06, \mathrm{BF}_{10}$ $=0.618$, or the neutral-blank group, $F(1,56)=2.11, p=.152, \eta_{p}{ }^{2}=.04, \mathrm{BF}_{10}=0.389$. Causal Ratings Test

The causal ratings showed a similar pattern to the prediction test ratings, and were analyzed in the same way, using factorial ANOVAs and simple effects. When comparing A with $\mathrm{C}$, the ANOVA revealed a main effect of stimulus, $F(1,222)=$ 23.9, $p<.001, \eta_{p}{ }^{2}=.10, \mathrm{MSE}=268.3$, and a significant interaction between stimulus and indicator group, $F(1,222)=7.26, p=.008, \eta_{p}{ }^{2}=.03$, MSE $=268.3$. All other main effects and interactions were not significant, $F_{\mathrm{S}}<1.82$. The pattern of results was similar when comparing $\mathrm{B}$ with $\mathrm{D}$, with a significant main effect of stimulus, $F(1,222)=7.47, p=.007, \eta_{p}{ }^{2}=.03, \mathrm{MSE}=224.2$, and a significant interaction between stimulus and indicator group, $F(1,222)=12.6, p<.001, \eta_{p}{ }^{2}=.05, \mathrm{MSE}=$ 224.2 , and no other effects reaching significance, $F_{\mathrm{S}}<3.83, p \mathrm{~s}>.051$. In each case the stimulus $\times$ indicator group interaction reflected a greater difference in ratings between $\mathrm{A}$ and $\mathrm{C}$, or B and D, in the "?" groups than the blank groups.

We again conducted simple effect analyses with Holm-Bonferroni correction. For the causal ratings, the observed effects were comparable for A and B. Comparing $\mathrm{A}$ and $\mathrm{C}$ in each group separately, participants rated A significantly lower than $\mathrm{C}$ when the "?" was presented as the indicator of missing feedback, regardless of whether the neutral, $F(1,50)=15.6, p<.001, \eta_{p}{ }^{2}=.24, \mathrm{MSE}=279.3, \mathrm{BF}_{10}=101.1$, or $\mathrm{MrX}$ cover story, $F(1,56)=17.1, p<.001, \eta_{p}{ }^{2}=.23, \mathrm{MSE}=178.0, \mathrm{BF}_{10}=186.6$, was presented. In contrast, in both groups where the feedback was blank, there was no significant difference between $\mathrm{A}$ and $\mathrm{C}, F_{\mathrm{S}}<2.14$, BFs $<0.383$. Similarly, B was rated significantly higher than $\mathrm{D}$ in both the neutral-?, $F(1,50)=6.49, p=.014, \eta_{p}{ }^{2}=$ $.11, \mathrm{MSE}-240.4, \mathrm{BF}_{10}=2.80$, and MrX-? groups, $F(1,56)=7.85, p=.007, \eta_{p}{ }^{2}=.12$, $\mathrm{MSE}=355.5, \mathrm{BF}_{10}=4.89$, but not for the groups receiving blank feedback, $F_{\mathrm{s}}<1.36$, $\mathrm{BFs}<0.275$.

\section{Discussion}

The results of Experiment 4 suggest that the primary driver of the regressionto-baseline effect observed in Experiment 3 for cues presented without feedback in phase 2 was the use of a "?" symbol to indicate missing feedback. Experiment 4 
observed this regression-to-baseline effect both for the cue that was previously reinforced (A) and the cue that was previously non-reinforced (B) in groups for whom missing feedback was indicated with "'?", and no evidence of this effect in groups for whom the missing feedback was simply a blank screen. This effect was evident in the training phase where ratings to cue $\mathrm{A}$ decreased and ratings to cue $\mathrm{B}$ increased linearly over trials. There were also clear differences between the no-feedback cues and the control cues in the outcome prediction test and causal ratings test. Although the training data suggested a slight regression effect in the MrX-blank group, the simple effects at test were mostly null in this group. The results in the neutral-blank and MrX-? group provide a replication of the findings of Experiments 2 and 3 respectively in an independent sample of participants. In addition, Experiment 4 also revealed more reliable effects of the no-feedback manipulation with regards to cue $\mathrm{B}$, although the effects were still stronger for cue A (see Figure E2 in Supplemental Materials). Regardless, the observation of significant effects for both no-feedback cues provides support for the idea that withholding feedback results in a regression in ratings to the midpoint of the scale.

\section{General Discussion}

In this study, we examined the common assumption that presenting cues under no-feedback conditions in associative learning experiments is effective in preventing further learning that might contaminate subsequent test trials. Our results suggest that this assumption holds in some conditions but not others. Experiment 1 showed that instructing participants to expect no feedback about the outcomes prior to a test phase was effective in preventing learning during that test phase. Experiment 2 showed that instructing participants to expect no feedback on specific training trials (presented alongside cues with outcome feedback) was also successful in preventing learning. In contrast to these null effects, Experiment 3 showed that integrating the no-feedback instructions into the allergist cover story and presenting a "?" as a placeholder for the missing outcome feedback resulted in a degree of regression towards baseline responding in predictive judgements (the midpoint of the scale). This effect of nofeedback trials in training persisted to influence responding to target cues in the test and causal ratings phase. Experiment 4 replicated and extended Experiments 2 and 3 by manipulating both cover story (neutral vs. Mr X forgot to write down his allergic reactions) and missing feedback indicator (blank screen vs. "?” symbol) in a between- 
subjects factorial design. The results showed that the "?" feedback, rather than the cover story, was primarily responsible for the regression-to-baseline effect.

The key null effects from Experiments 1, 2, and 4 were found by comparing target cues presented without feedback during the critical second phase of the task, to control cues that were not presented during this phase. These findings were observed on both outcome predictive ratings and in causal ratings, were supported by Bayes Factors, and were exhibited by nearly all individuals (see Supplemental Materials). In contrast to the no-feedback cues, cues that were presented with explicit "no outcome" feedback (in Group Extinction of Experiment 1, cue E in Experiments 2-4) showed a typical extinction pattern, demonstrating that this procedure was able to detect changes in predictive judgements during phase 2 - which makes the null effect of the no-feedback manipulation more diagnostic.

We noted in the Introduction that the no-feedback approach was developed with the aim of allowing researchers to measure participants' responding to particular cues while preventing further learning about those cues from occurring. Taken together, the results of the current study suggest that the ideal way to administer nofeedback trials in this regard is to use neutral instructions informing participants to expect feedback to be withheld, and avoid presenting alternative feedback in place of information about the outcome. Experiment 1 showed that the typical method of presenting test trials in the absence of any feedback (i.e., going straight to a blank inter-trial interval after participants respond) was successful in preventing learning from occurring. Experiments 2 and 4 showed that retaining the feedback period but simply presenting a blank screen in place of the outcome feedback also prevented learning.

In contrast, Experiments 3 and 4 showed that use of a "?" to indicate missing feedback had marked effects on subsequent test ratings. The "?" feedback may have highlighted participants' lack of knowledge about the outcome and made them uncertain, causing a regression towards the midpoint of the response scale in response to this uncertainty. Alternatively, the "?" could have functioned as another outcome which competed with the previous associations learned in the acquisition phase, and/or interfered with the memory of those associations. Although overall, the effect of withholding feedback appeared to be stronger for a cue that was previously reinforced (cue A) compared to a cue that was previously non-reinforced (cue B), Experiment 4 demonstrated reliable effects for both of these cues. One explanation for 
the asymmetry in effect size could be that the "?" feedback was perceived by participants to be more similar to the explicit "no outcome" feedback than to "allergic reaction" feedback, such that prediction error was greater when a previously reinforced cue (relative to a non-reinforced cue) was now paired with "?” feedback. If the regression effect is partly determined by this prediction error, then larger effects would be observed for the no-feedback cues that were previously reinforced compared to those that were not reinforced.

A further possibility is that the "?" feedback encouraged participants to think about what the missing outcomes might be. This idea that participants sometimes treat missing feedback as informative is consistent with related findings from multiple-cue judgement tasks, where participants' behavior suggests that rather than discounting trials with missing feedback, they infer the most likely outcome from prior knowledge; a phenomenon termed constructivist coding (Elwin et al., 2007; Henriksson et al., 2010). Although in our task, the most likely outcomes would arguably consist of the cue-outcome associations presented in the first phase of training, the presentation of the "?" may have led participants to infer that the cueoutcome associations had changed. This is conceivable since in Experiments 2-4, the onset of the missing feedback was accompanied by a change in outcome for the extinguished cue (E). If participants assumed that the outcomes for the no-feedback cues had also changed, then this would explain the regression-to-baseline effects observed in Experiments 3 and 4. Future studies could investigate whether participants actively construct reasons for missing outcomes and what factors dictate inferences about the identity of the censored outcomes.

The nature of the instructions providing a cover story for when and why feedback would be omitted had a much weaker influence on regression-to-baseline effects. Nevertheless, we advise against embedding the explanation for the missing feedback in the cover story of the causal scenario for a number of reasons. In the MrX-blank group of Experiment 4, ratings for cue A (but not for cue B) showed some evidence of a linear decrease over training trials (see Figure 4a). Although the key comparisons were not reliable at test, it is possible that they could have been with a larger sample, or with additional no-feedback trials. We also found some differences between cover-story conditions in the distribution of responses in a post-experimental question asking participants to explain why they thought feedback was withheld (see Supplemental Materials). Participants given neutral instructions were more likely to 
report not thinking too much about why the outcome feedback was withheld, compared to participants for whom the missing feedback was attributed to $\mathrm{Mr} \mathrm{X}$. In summary, our data suggest that the ideal way to administer the no-feedback testing procedure is to use neutral instructions. This will help ensure that participants know that the missing feedback is part of the experiment, and reduce the likelihood that they construct alternative explanations or think about the identity of the censored outcomes.

Our results were obtained with a single predictive/causal learning task, using relatively simple designs. It would be valuable to test the generality of our findings in other tasks, in particular conditioning procedures with a biologically salient outcome such as electric shock. However, we consider that our results are likely to be applicable to a wide range of tasks used in cognitive psychology such as category learning, statistical learning, sequence learning, and judgement and decision-making tasks. From a theoretical perspective, our results are informative in confirming that participants are capable of ignoring information (the absence of outcomes) that would otherwise be treated as relevant within the learning task, provided the reason for that omission is explained clearly. More broadly, they also highlight the importance of considering participants' reasoning and interpretational processes in learning; the same experiences can lead to different learning outcomes depending on prior knowledge (Kemp \& Tenenbaum, 2009) and assumptions about the evidence being presented (Griffiths \& Tenenbaum, 2001).

In conclusion, our study demonstrates that common methods of testing with no feedback can be used without concern of contaminating later test trials in predictive/causal learning experiments. These findings suggest that researchers should implement the procedure in such a way that the absence of feedback is attributed to the experimenter, and most importantly, avoid highlighting the absence of the relevant information through presentation of alternative feedback. 
26 Nonreactive testing

\section{Open Practices Statement}

The data and materials for all experiments are available at https://osf.io/dvn9g/. None of the experiments were preregistered. 


\section{References}

Cohen J. (1988). Statistical Power Analysis for the Behavioral Sciences. New York, NY: Routledge Academic.

Collins, D. J., \& Shanks, D. R. (2006). Summation in causal learning: Elemental processing or configural generalization. The Quarterly Journal of Experimental Psychology, 59(9), 1524-1534. https://doi.org/10.1080/17470210600639389

de Leeuw, J. R. (2015). jsPsych: A JavaScript library for creating behavioral experiments in a web browser. Behavior Research Methods, 47(1), 1-12. https://doi.org/10.3758/s13428-014-0458-y

Dickinson, A., Shanks, D., \& Evenden, J. (1984). Judgement of act-outcome contingency: The role of selective attribution. The Quarterly Journal of Experimental Psychology, 36A(1), 29-50.

Dunsmoor J. E., \& LaBar, K. S. (2013). Effects of discrimination training on fear generalization gradients and perceptual classification in humans. Behavioral Neuroscience, 127(3), 350-356. http://dx.doi.org/10.1037/a0031933

Eatherington, C. J., \& Haselgrove, M. (2020). The role of prediction in learned predictiveness. PsyArXiv. https://doi.org/10.31234/osf.io/wqhz2

Elwin, E., Juslin, P., Olsson, H., \& Enkvist, T. (2007). Constructivist coding: Learning from selective feedback. Psychological Science, 18(2), 105-110. https://doi.org/10.1111/j.1467-9280.2007.01856.x

George, D. N., \& Kruschke, J. K. (2012). Contextual modulation of attention in human category learning. Learning and Behavior, 40, 530-541. https://doi.org/ $10.3758 / \mathrm{s} 13420-012-0072-8$

Graham, S., Jie, H. L., Chan, H., McLaren, I. P. L., \& Wills, A. (2011). Simultaneous backward conditioned inhibition and mediated conditioning. Journal of Experimental Psychology: Animal Behavior Processes, 37(2), 241-245. https://doi.org/10.1037/a0021828

Gluck, M. A., \& Bower, G. H. (1988). From conditioning to category learning: An adaptive network model. Journal of Experimental Psychology: General, 117(3), 227-247. https://doi.org/10.1037/0096-3445.117.3.227

Henriksson, M. P., Elwin, E., \& Juslin, P. (2010). What is coded into memory in the absence of outcome feedback? Journal of Experimental Psychology: 
Learning, Memory, and Cognition, 36(1), 1-16.

https://doi.apa.org/doi/10.1037/a0017893

Holm, S. (1979). A simple sequentially rejective multiple test procedure.

Scandinavian Journal of Statistics, 6(2), 65-70.

https://www.jstor.org/stable/4615733

Honig, W., \& Urcuioli, P. J. (1981). The legacy of Guttman \& Kalish (1956): 25 years of research on stimulus generalization. Journal of the Experimental Analysis of Behavior, 36(3), 405-445. https://doi.org/10.1901/jeab.1981.36405

Humphreys, L. G. (1939). The effect of random alternation of reinforcement on the acquisition and extinction of conditioned eyelid reactions. Journal of Experimental Psychology, 25, 141, 158. http://dx.doi.org/10.1037/h0058138

Jeffreys, H. (1939). Theory of probability (1st ed.). Oxford, UK: Oxford University Press.

Kemp, C., \& Tenenbaum, J. B. (2009). Structured statistical models of inductive reasoning. Psychological Review, 116(1), 20-58. https://doi.org/10.1037/a0014282

Lange, K., Kühn, S., \& Filevich, E. (2015). "Just Another Tool for Online Studies" (JATOS): An easy solution for setup and management of web servers supporting online studies. PLoS ONE, 10(6): e0130834. https://doi.org/10.1371/journal.pone.0130834

Le Pelley M. E., \& McLaren, I. P. L. (2001). Retrospective revaluation in humans: Learning or memory? The Quarterly Journal of Experimental Psychology B, 54(4), 311-352. https://doi.org/10.1080\%2F713932762

Le Pelley M. E., \& McLaren, I. P. L. (2004). Associative history affects the associative change undergone by both presented and absent cues in human causal learning. Journal of Experimental Psychology: Animal Behavior Processes, 30(1), 67-73. https://doi.org/10.1037/0097-7403.30.1.67

Le Pelley, M. E., Griffiths, O., \& Beesley, T. (2017). Associative accounts of causal cognition. In M. R. Waldmann (Ed.), The Oxford handbook of causal reasoning, (pp. 13-28). Oxford University Press. https://doi.org/ 10.1093/oxfordhb/9780199399550.013.2 
Lee, J. C., Hayes, B. K., \& Lovibond, P. F. (2018). Peak shift and rules in human generalization. Journal of Experimental Psychology: Learning, Memory, and. Cognition, 44(12), 1955-1970. https://doi.org/10.1037/xlm0000558

Lee J. C., \& Livesey, E. J. (2012). Second-order conditioning and conditioned inhibition: Influences of speed versus accuracy on human causal learning. PLoS ONE, 7(11), e49899. https://doi.org/10.1371/journal.pone.0049899

Lee J. C., \& Lovibond, P. F. (in press). Individual differences in causal structures inferred during feature negative learning. Quarterly Journal of Experimental Psychology, 74(1), 150-165. https://doi.org/10.1177/1747021820959286

Lee, J. C., Lovibond, P. F., Hayes, B. K., \& Navarro, D. J. (2019). Negative evidence and inductive reasoning in generalization of associative learning. Journal of Experimental Psychology: General, 148(2), 289-303. https://doi.org/10.1037/xge0000496

Lenth, R. (2019). emmeans: Estimated Marginal Means, aka Least-Squares Means. R package version 1.4.2. https://CRAN.R-project.org/package=emmeans

Livesey, E. J., Don, H. J., Uengoer, M., \& Thorwart, A. (2019). Transfer of associability and relational structure in human associative learning. Journal of Experimental Psychology: Animal Learning and Cognition, 45, 125-142. https://doi.org/10.1037/xan0000197

Luque, D., Beesley, T., Morris, R. W., Jack, B. N., Griffiths, O., Whitford, T. J., \& Le Pelley, M. E. (2017). Goal-directed and habit-like modulations of stimulus processing during reinforcement learning. The Journal of Neuroscience, 37(11), 3009-3017. https://doi.org/10.1523/JNEUROSCI.3205-16.2017

Matute, H., Arcediano, F., \& Miller, R. R. (1996). Test question modulates cue competition between causes and between effects. Journal of Experimental Psychology: Learning, Memory, and Cognition, 22(1), 182-196. https://doi.org/10.1037/0278-7393.22.1.182

Morey R. D., \& Rouder, J. N. (2018). BayesFactor: Computation of Bayes Factors for Common Designs. R package version 0.9.12-4.2. https://CRAN.Rproject.org/package $=$ BayesFactor

Mowrer, O. H., \& Jones, H. (1945). Habit strength as a function of the pattern of reinforcement. Journal of Experimental Psychology, 35, 293-311. http://dx.doi.org/10.1037/h0056678 
R Core Team (2019). R: A language and environment for statistical computing. R Foundation for Statistical Computing, Vienna, Austria. https://www.Rproject.org/.

Rescorla, R. A. \& Wagner, A. R. (1972). A theory of Pavlovian conditioning: Variations on the effectiveness of reinforcement and non-reinforcement. In A. H. Black \& W. F. Prokasy (ed.), Classical conditioning II: Current research and theory (pp. 64-99). Appleton-Century-Crofts.

Shanks D. R., \& Darby, R. J. (1998). Feature- and rule-based generalization in human associative learning. Journal of Experimental Psychology: Animal Behavior Processes, 24(4), 405-415. https://doi.org/10.1037/0097-7403.24.4.405

Shanks, D. R., \& Dickinson, A. (1987). Associative accounts of causality judgment. In G. H. Bower (Ed.), The psychology of learning and motivation: Advances in research and theory, Vol. 21 (pp. 229-261). Academic Press.

Singmann, H., Bolker, B., Westfall, J., Aust, F., \& Ben-Shachar, M. S. (2019) afex: Analysis of Factorial Experiments. R package version 0.25-1. https://CRAN.R-project.org/package=afex

Tenenbaum, J. B., \& Griffiths, T. L. (2001). Generalization, similarity, and Bayesian inference. Behavioral and Brain Sciences, 24, 629-640. https://doi.org/10.1017/s0140525x01000061

Uengoer, M., Lachnit, H., \& Pearce, J. M. (2020). The role of common elements in the redundancy effect. Journal of Experimental Psychology: Animal Learning and Cognition, 46(3), 286-296. http://doi.org/10.1037/xan0000236

Wasserman, E. A. (1990). Attribution of causality to common and distinctive elements of compound stimuli. Psychological Science, 1(5), 298-302. https://doi.org/10.1111/j.1467-9280.1990.tb00221.x

Wong, A. H. K., \& Lovibond, P. F. (2017). Rule-based generalization in single-cue and differential fear conditioning in humans. Biological Psychology, 129, 111120. https://doi.org/ 10.1016/j.biopsycho.2017.08.056

Williams, D. A., Sagness, K. E. \& McPhee, J. E. (1994). Configural and elemental strategies in predictive learning. Journal of Experimental Psychology: Learning, Memory and Cognition, 20(3), 694-709. https://doi.org/10.1037/0278-7393.20.3.694

Wills, A., Lavric, A., Croft, G. S., \& Hodgson, T. L. (2007). Predictive learning, prediction errors, and attention: Evidence from event-related potentials and 
eye tracking. Journal of Cognitive Neuroscience, 19(5), 843-854.

https://doi.org/10.1162/jocn.2007.19.5.843

Wills, A J., Lavric, A., Hemmings, Y., \& Surrey, E. (2014). Attention, predictive learning, and the inverse base-rate effect: Evidence from event-related potentials. NeuroImage, 87, 61-71.

https://doi.org/10.1016/j.neuroimage.2013.10.060 